\title{
Orbital angular momentum interference of trapped matter waves
}

\author{
Filip Kiałka, ${ }^{1,2}$ Benjamin A. Stickler, ${ }^{3,2}$ and Klaus Hornberger $\circledast^{2}$ \\ ${ }^{1}$ Faculty of Physics, University of Vienna, Boltzmanngasse 5, A-1090 Vienna, Austria \\ ${ }^{2}$ Faculty of Physics, University of Duisburg-Essen, Lotharstraße 1, 47048 Duisburg, Germany \\ ${ }^{3}$ Quantum Optics and Laser Science, Imperial College London, SW72AZ London, United Kingdom
}

(Received 7 November 2019; revised manuscript received 9 April 2020; accepted 15 April 2020; published 6 May 2020; corrected 19 March 2021)

\begin{abstract}
We introduce a matter wave interference scheme based on the quantization of orbital angular momentum in a ring trap. It operates without beam splitters, is sensitive to geometric phases induced by external gauge fields, and allows measuring interatomic scattering lengths. We argue that orbital angular momentum interferometry offers a versatile platform for quantum coherent experiments with cold atoms and Bose-Einstein condensates using state-of-the-art technology.
\end{abstract}

DOI: 10.1103/PhysRevResearch.2.022030

Introduction. Trapped interference experiments [1-8] are promising platforms for the next generation of force and acceleration sensors. Guiding matter waves enables atom interferometers with long interrogation times, while providing considerable freedom for choosing the geometry [9-12]. Toroidal traps are particularly attractive for fundamental quantum experiments [13-18] and for precision sensing [19-21] with ultracold gases or fluids. The ring geometry implies that the orbital angular momentum of the revolving particles is conserved. As argued in the following, its fundamental quantization can be exploited to realize trapped interference schemes requiring no beam splitters.

We note that the free quantum dynamics in a ring geometry exhibit quantum revivals. An initially well-localized wave packet quickly disperses along the ring on a timescale determined by the orbital angular momentum spread. Only after a much longer quantum revival time, which is independent of the initial state, does the localized wave packet briefly reappear due to the quantization of orbital angular momentum [22]. Similar revival effects are encountered in the orientation of revolving molecules [23-25], and they have been proposed for electromagnetic pulse shaping in semiconductors [26] as well as for macroscopic quantum superposition tests with nanorotors [27].

Here, we propose an interference scheme which exploits the brief emergence of a balanced superposition at half the revival time. By imprinting a relative phase on the superposition, one can coherently control at which antipode the wave packet reappears after the full revival time. The presence of an additional gauge field induces a rotation of the revival determined by the accumulated geometric phase. In contrast to many existing proposals for interference in ring

Published by the American Physical Society under the terms of the Creative Commons Attribution 4.0 International license. Further distribution of this work must maintain attribution to the author(s) and the published article's title, journal citation, and DOI. traps [19,28,29], orbital angular momentum interference does not rely on atomic spin states or collective excitations. It is thus applicable to all matter-wave experiments with a toroidal geometry, ranging from electrons in solid state quantum rings [30] to nanoparticles in optomechanical traps [31]. Here we discuss the special case of optically trapped atomic clouds or Bose-Einstein condensates (BECs), and show that this scheme is sufficiently resilient to be realizable with state-ofthe-art technology.

Interference scheme. In order to explain the interference scheme we first consider the idealized case of a point particle of mass $m$ confined to a circle of radius $R$. Its Hamiltonian reads $\mathrm{H}=\mathrm{L}_{z}^{2} / 2 m R^{2}$. Since the eigenvalues of the orbital angular momentum operator $L_{z}$ are integer multiples of $\hbar$, with eigenstates $|\ell\rangle$, the time evolution operator $\mathrm{U}_{0}(t)=$ $\sum_{\ell \in \mathbb{Z}} \exp \left(-i \hbar t \ell^{2} / 2 m R^{2}\right)|\ell\rangle\langle\ell|$ is unity for all even multiples of the revival time

$$
T_{\mathrm{rev}}=\frac{2 \pi m R^{2}}{\hbar} .
$$

A straightforward calculation shows that the evolution for the revival time performs a $\pi$ rotation, $\mathrm{U}_{0}\left(n T_{\text {rev }}\right)=$ $\exp \left(i n \pi \mathrm{L}_{z} / \hbar\right)$, with $n \in \mathbb{N}_{0}$. In a similar fashion, free evolution for $T_{\text {rev }} / 2$ acts as a beam splitter, preparing a balanced superposition of the initial state and its $\pi$-rotated version $[24,32]$,

$$
\mathrm{U}_{0}\left(\frac{T_{\mathrm{rev}}}{2}\right)=\frac{e^{-i \pi / 4}}{\sqrt{2}}\left(\mathbb{1}+i e^{i \pi \mathrm{L}_{z} / \hbar}\right),
$$

where $\mathbb{1}$ is the unity operator.

An initially tightly confined wave packet thus first disperses on a short timescale determined by its initial angular momentum uncertainty. The state then remains delocalized over the ring for most of time, showing fractional revivals such as Eq. (2) at fractions of the revival time. The lifetime of these fractional and full revivals is determined by the initial dispersion time, and is thus typically orders of magnitude smaller than the revival time itself. 
(a)

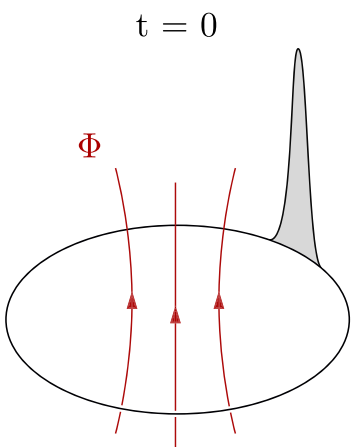

(c)

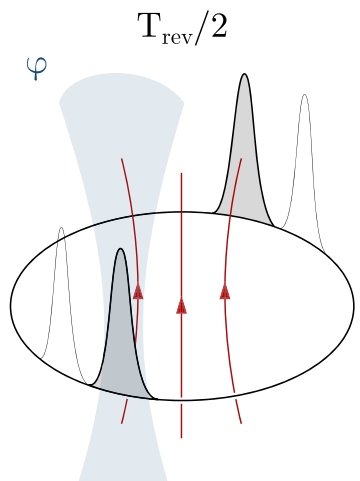

(b)

$\mathrm{t} \ll \mathrm{T}_{\text {rev }}$

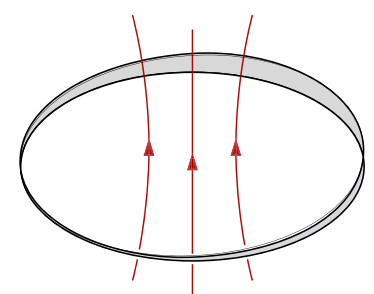

(d)

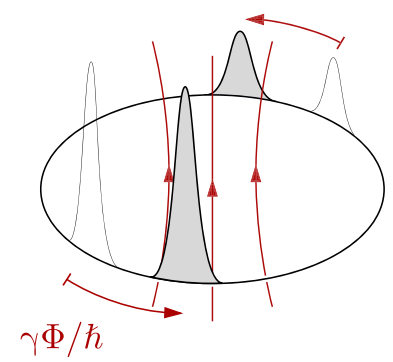

FIG. 1. Schematic illustration of the orbital angular momentum interference effect. A localized wave packet (a) quickly disperses (b), before reappearing (c) at $T_{\text {rev }} / 2$ for a short period of time in a balanced superposition of the original and mirrored locations; see (1) and (2). Applying a relative phase $\varphi$ between the two superposition components controls final population imbalance at the antipodes (d) after further time evolution for $T_{\text {rev }} / 2$. If a gauge field is turned on quickly after releasing the wave packet, the interference pattern (black) is rotated with respect to the field-free case (gray) by an angle $\gamma \Phi / \hbar$, equal to the Aharonov-Bohm phase [see Eq. (3)].

The dynamical beam splitting described by (2) is exploited by the following interference scheme; see Fig. 1(a): The particle is initially prepared in a well-localized state $\left|\psi_{0}\right\rangle$. After dispersing on a short timescale, the localized state reappears at half of the revival time in a balanced superposition $\left(\left|\psi_{0}\right\rangle+i\left|\psi_{\pi}\right\rangle\right) / \sqrt{2}$, with the $\pi$-rotated initial state $\left|\psi_{\pi}\right\rangle=$ $\exp \left(i \pi \mathrm{L}_{z} / \hbar\right)\left|\psi_{0}\right\rangle$. Then a relative phase $\varphi$ is induced between the two wave packets, for instance gravitationally by tilting the ring, optically via laser illumination, or in the case of an atomic cloud via magnetic control of the scattering length. After imprinting the phase, the state evolves freely for another $T_{\text {rev }} / 2$, yielding the final state $\left|\psi_{f}\right\rangle=\cos (\varphi / 2)\left|\psi_{\pi}\right\rangle+$ $i \sin (\varphi / 2)\left|\psi_{0}\right\rangle$. The final position of the particle is thus determined interferometrically.

Gauge fields and external potentials. The interference effect depends sensitively on the interaction with external gauge fields. If the field $\mathbf{A}(\mathbf{r})$ is minimally coupled to the canonical angular momentum $L_{z}$, the kinetic angular momentum is $\mathrm{L}_{z}-\gamma R A(\hat{\alpha})$. Here $\gamma$ is the gauge coupling and $A(\alpha)=$ $\mathbf{A}\left(\operatorname{Re}_{\rho}(\alpha)\right) \cdot \mathbf{e}_{\alpha}(\alpha)$ is the azimuthal component of the gauge field evaluated at the angular position $\alpha$.

The presence of $\mathbf{A}(\mathbf{r})$ implies a gauge-invariant flux $\Phi=R \oint d \alpha A(\alpha)$ piercing the ring interferometer and thus modifying the free time evolution of the matter wave. The

unitary time evolution operator becomes

$$
\mathrm{U}_{\Phi}(t)=\mathrm{V} \exp \left(i \frac{\gamma \Phi}{\hbar} \frac{t}{T_{\text {rev }}} \frac{\mathrm{L}_{z}}{\hbar}\right) \mathrm{U}_{0}(t) \mathrm{V}^{\dagger},
$$

where $\mathrm{V}=\exp \left(-i \gamma \Phi \hat{\alpha} / 2 \pi \hbar+i \gamma R / \hbar \int_{0}^{\hat{\alpha}} d \alpha^{\prime} A\left(\alpha^{\prime}\right)\right)$ can always be set to unity by choosing an appropriate gauge (symmetric gauge in the case of a constant field). Thus, a finite flux induces a rotation of the recurred wave packet by the Aharanov-Bohm-type phase $\gamma \Phi / \hbar$.

For example, if the particles are electrically charged, $\gamma=$ $q$, a magnetic flux $\Phi$ through the ring will shift the energy levels $[32,33]$ causing the wave packet to rotate. In a similar fashion, the Aharonov-Casher phase [34] can be measured if a magnetic dipole $\mathbf{m}=m_{0} \mathbf{e}_{z}$ evolves in presence of the electrostatic field $\mathbf{E}\left(R \mathbf{e}_{\rho}\right)=E_{0} \mathbf{e}_{\rho}$ produced by a line charge. In this case one has $\gamma \mathbf{A}=\mathbf{m} \times \mathbf{E} / c^{2}$, implying $\gamma \Phi=2 \pi R E_{0} m_{0} / c^{2}$. Likewise, geometric phases can result for a permanent or induced electric dipole $\mathbf{p}$ in a magnetostatic field $\mathbf{B}$, so that $\gamma \mathbf{A}=\mathbf{p} \times \mathbf{B}$ [35], or for a massive particle in a noninertial frame rotating with angular frequency $\omega$ around the trap center, so that $\gamma \mathbf{A}=m R^{2} \boldsymbol{\omega}$.

The presence of a weak external potential $V(\alpha)=$ $V_{0} \cos \left(\alpha-\alpha_{0}\right)$, such as that arising from a constant tilt of the ring, leads to phase dispersion. To leading order in $V_{0}$, the energies are shifted by

$$
\Delta E_{\ell}^{(\mathrm{pot})} \approx \frac{m R^{2} V_{0}^{2}}{4 \hbar^{2}}\left(\ell^{2}-\frac{1}{4}\right)^{-1} .
$$

Since this is not proportional to $\ell^{2}$, a conservative torque affects the shape of the recurring wave packet. This is in contrast to gauge fields, which only shift the position of the revival.

Revivals in $3 D$ torus traps. The evolution of a particle in a real-world (three-dimensional) torus trap differs from the idealized situation described so far. The dynamics transverse to the ring tangent affect the angular dynamics even if the transverse motion remains in its ground state, since the centrifugal force distorts the level spacing. Shape imperfections and excitations of the transverse degrees of freedom can further affect the interference. We will show next that the proposed orbital angular momentum interference protocol is nevertheless surprisingly robust and remains feasible for realistic trap geometries.

To study the dynamics in a real-world torus trap, we expand the full 3D Hamiltonian of a particle in a torus trap and consider leading-order corrections in the transverse size of the wave packet. For this sake, we use a FrenetSerret coordinate system $(s, u, v)$ with arc length $s$ and two transverse coordinates $u, v$. Thus, the position vector is $\mathbf{r}=$ $\mathbf{R}(s)+u \mathbf{n}(s)+v \mathbf{b}(s)$, where $\mathbf{R}(s)$ traces the center line of the torus trap, while $\mathbf{n}(s)=\mathbf{R}^{\prime \prime}(s) / \kappa$ and $\mathbf{b}(s)=\mathbf{R}^{\prime}(s) \times \mathbf{n}(s)$ span the transverse plane at each position [36,37]. Here, $\kappa=$ $\left|\mathbf{R}^{\prime \prime}(s)\right|$ is the curvature, where prime denotes derivative with respect to $s$.

Since the new coordinate system $(s, u, v)$ is curved, coordinate-space normalization of the wave function includes the root of the metric determinant (Jacobian) $h$. Expressing the latter as $h=1-\kappa u$ and assuming that the trapping potential is separable in the transverse direction yields the Hamilto- 
nian $[36,37]$

$$
\begin{aligned}
\mathrm{H}_{s}= & -\frac{\hbar^{2}}{2 m}\left[\partial_{s} \frac{\partial_{s}}{h^{2}}+\partial_{u}^{2}+\partial_{v}^{2}+\frac{\kappa^{2}}{4 h^{2}}+\frac{5\left(h^{\prime}\right)^{2}}{4 h^{4}}-\frac{h^{\prime \prime}}{2 h^{3}}\right] \\
& +V_{u}(u)+V_{v}(v),
\end{aligned}
$$

which acts on the rescaled wave function $\chi=\sqrt{h} \psi$.

If the radially confining potential is harmonic with frequency $\omega_{\perp}$ and assuming that centrifugal distortions and small deviations from the ideal circular trap can be described by expanding the Hamiltonian to first order in the small quantities $\kappa \sigma_{u}, \kappa^{\prime} \sigma_{u} / \kappa$, and $\kappa^{\prime \prime} \sigma_{u} / \kappa^{2}$ (with $\sigma_{u}=\sqrt{\hbar / m \omega_{\perp}}$ the width of the transverse ground state),

$$
\begin{aligned}
\mathrm{H}_{s} \approx & -\frac{\hbar^{2}}{2 m}\left[(1+2 \kappa u)\left(\partial_{s}^{2}+\frac{\kappa^{2}}{4}\right)+2 \kappa^{\prime} u(1+3 \kappa u) \partial_{s}\right. \\
& \left.+\frac{\kappa^{\prime \prime} u}{2}+\partial_{u}^{2}+\partial_{v}^{2}\right]+\frac{m \omega_{\perp}^{2}}{2} u^{2}+V_{v}(v) .
\end{aligned}
$$

Centrifugal energy corrections. For an ideal torus where $\kappa=1 / R$ the stationary Schrödinger equation becomes separable. It admits solutions of the form

$$
\chi_{\ell k n}(s, u, v)=\frac{1}{\sqrt{2 \pi R}} e^{i \ell s / R} \xi_{\ell k}(u) \Psi_{n}(v),
$$

with eigenenergies $E_{\ell k n}=\hbar^{2} \ell^{2} / 2 m R^{2}+E_{\ell k}^{(u)}+E_{n}^{(v)}$ where $k, n \in \mathbb{N}_{0}$. Here, $\Psi_{n}(v)$ are normalized eigenstates of the harmonic motion out of the ring plane, whose eigenenergies $E_{n}^{(v)}$ are independent of $\ell$ and thus do not affect the revival structure of the matter wave.

The radially confining harmonic potential in the Schrödinger equation for $\xi_{\ell k}(u)$ is centrifugally shifted by $u_{\ell}=\hbar^{2}\left(\ell^{2}-1 / 4\right) / m^{2} \omega_{\perp}^{2} R^{3}$,

$$
\left[-\frac{\hbar^{2}}{2 m} \partial_{u}^{2}+\frac{m \omega_{\perp}^{2}}{2}\left(u^{2}+2 u u_{\ell}\right)\right] \xi_{\ell k}(u)=E_{\ell k}^{(u)} \xi_{\ell k}(u) .
$$

Thus, the eigenergies

$$
E_{\ell k}^{(u)}=\hbar \omega_{\perp}\left(k+\frac{1}{2}\right)-\frac{\hbar^{4}}{2 m^{3} \omega_{\perp}^{2} R^{6}}\left(\ell^{2}-\frac{1}{4}\right)^{2}
$$

are lowered due to the centrifugal barrier.

The $\ell$ dependence in the eigenenergies (9) can shift and diminish the revival. Specifically, the $\ell^{2}$ term in Eq. (9) delays the revival without affecting its visibility, while the $\ell^{4}$ correction decreases the fidelity of the revival and may further modify the revival time. The optimal recurrence time can be determined numerically from this equation.

Shape imperfections. In practice, deviations from the perfect circular shape of the torus trap are the most important source of imperfections for optical traps. In particular, residual astigmatism in the focusing optics may introduce a finite ellipticity to the trap, which can be quantified with the help of (6).

We replace the arc length with the eccentric anomaly $\beta \in$ $[-\pi, \pi)$ used for the standard parametrization of the ellipse. Thus, $\partial_{s}=h_{\varepsilon}^{-1}(\beta) \partial_{\beta} / R$, where $R$ and $\varepsilon$ are the semimajor axis and the eccentricity and $h_{\varepsilon}(\beta)=\sqrt{1-\varepsilon^{2} \cos ^{2} \beta}$ is the Jacobi determinant of the ellipse. In lowest order of $\varepsilon$, the Hamiltonian reads as $\mathrm{H}_{\beta}=h_{\varepsilon}^{1 / 2} \mathrm{H}_{s} h_{\varepsilon}^{-1 / 2} \approx \mathrm{H}_{\beta}^{(0)}+\varepsilon^{2} \mathrm{H}_{\beta}^{(\varepsilon)}$, where $\mathrm{H}_{\beta}^{(0)}$ describes the motion on the circle and

$$
\begin{aligned}
\mathrm{H}_{\beta}^{(\varepsilon)}= & -\frac{\hbar^{2}}{4 m R^{2}}\left[1+\frac{3 u}{R}+\left(1+\frac{5 u}{R}\right) \cos (2 \beta)\right] \partial_{\beta}^{2} \\
& +\frac{\hbar^{2} \sin (2 \beta)}{2 m R^{2}}\left(1+\frac{5 u}{R}+\frac{9 u^{2}}{R^{2}}\right) \partial_{\beta} \\
& -\frac{\hbar^{2}}{16 m R^{2}}\left[1+\frac{3 u}{R}-\left(1+\frac{11 u}{R}\right) \cos (2 \beta)\right] .
\end{aligned}
$$

This implies that the eccentricity-induced energy shift reads in first-order perturbation theory

$$
\Delta E_{\ell}^{(\varepsilon)}=\frac{\hbar^{2} \varepsilon^{2}}{8 \pi m R^{2}}\left(1+\frac{3 u_{\ell}}{R}\right)\left(\ell^{2}-\frac{1}{4}\right) .
$$

Here we expressed the position expectation value of the radial state by the centrifugal shift of the harmonic potential (8), $\langle u\rangle=-u_{\ell}$. The first-order influence of a finite eccentricity is thus to decrease the revival time, while further diminishing the revival due to the $\ell$ dependence of the radial potential minimum $u_{\ell}$.

Implementation with BECs. We are now in a position to argue that the orbital angular momentum interference scheme can be realistically carried out with weakly interacting BECs in an optical torus trap. For concreteness, we consider a condensate of ${ }^{39} \mathrm{~K}$ in a trap formed by two coaxial Gaussian beams, one repulsive and one attractive, intersected with an attractive light sheet, as in Ref. [19]. The wavelengths of the red- and blue-detuned laser beams are assumed to be 830 and $532 \mathrm{~nm}$, respectively, with powers of 2 and $2.5 \mathrm{~mW}$ as well as waists of 13 and $5.5 \mu \mathrm{m}$. The light sheet with the same wavelength as the red-detuned laser has a power of $10 \mathrm{~mW}$ and waists of 5 and $200 \mu \mathrm{m}$, so that the trap radius is $R \approx 5.9 \mu \mathrm{m}$ and the transverse confining frequency $\omega_{\perp} \approx$ $6.4 \mathrm{kHz}$. The necessary coherence time of $T_{\mathrm{rev}} \approx 135 \mathrm{~ms}$ is experimentally within reach [8].

Figure 2 shows the simulated dynamics of the orbital angular momentum interference protocol for (a) a noninteracting and (b) a weakly interacting BEC of $N=2 \times 10^{4}{ }^{39} \mathrm{~K}$ atoms. We assume in both cases that the Feshbach resonances of ${ }^{39} \mathrm{~K}[38]$ are used to make the interactions (a) negligibly small or (b) equivalent to a scattering length of one Bohr radius. The tightly confined initial wave packet, loaded from threedimensional harmonic trap of frequency $\omega_{\perp}$, quickly disperses around the torus. It then reappears in a superposition after approximately $65 \mathrm{~ms}$. The presence of interactions diminishes the revival signal. However, even at a realistic transverse confinement and interaction strength, the effect is still clearly visible in the population imbalance displayed in panel (c). The latter shows that the interference visibility exhibits almost the ideal dependence on the imprinted phase. The numerical calculations are based on the Trotter-Suzuki expansion [39-41].

For this setup, the centrifugal energy shift (9) amounts to a few percent of the rotational energy for the highest-populated $\ell$ eigenstates $(\ell \simeq 25)$. The corresponding correction to the revival time is at a permille level, but, given the quick dispersion time, exact timing on the scale of a few microseconds is required to imprint the phase and to observe the revival. 

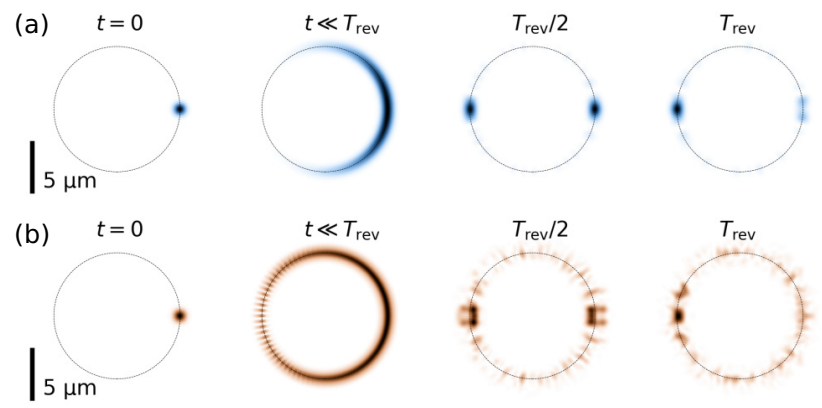

(c)

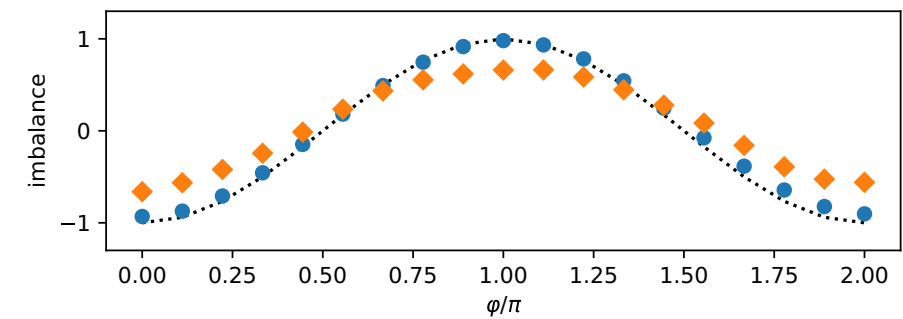

FIG. 2. Mean-field simulation of the interference scheme shown in Fig. 1(a) realized with a BEC of ${ }^{39} \mathrm{~K}$ in an optical trap. (a) Snapshots of the time evolution for a noninteracting condensate: initial particle density, dispersion, recurrent superposition at half of the revival time, and final interferometrically controlled revival with $\varphi=\pi / 3$. The external phase of $\exp \left(i \varphi \cos ^{2} \alpha\right)$ is applied on the left part of the ring at $T_{\text {rev }} / 2$. The revival time $T_{\text {rev }} \approx 135.8 \mathrm{~ms}$ is found by maximizing the overlap between the initial and final states for $\varphi=0$. (b) As in (a) but with interatomic interactions characterized by the scattering length of one Bohr radius for a BEC of $N=2 \times 10^{4}$ atoms. As a result of the interactions the revival time changes to $T_{\mathrm{rev}} \approx 136.2 \mathrm{~ms}$. (c) Interference signal as a function of external phase $\varphi$ in the noninteracting [as in (a), circles] and interacting [as in (b), diamonds] cases, as compared to the ideal situation (dotted line). The population imbalance is defined as $\left(N_{\mathrm{R}}-N_{\mathrm{L}}\right) /\left(N_{\mathrm{R}}+N_{\mathrm{L}}\right)$, where $N_{\mathrm{R}}, N_{\mathrm{L}}$ are the numbers of atoms on the right and left sides of the ring, weighted with $\cos ^{2} \alpha$.

In a similar fashion, the corrections of the revival time due to interactions must be accounted for, as has been done numerically in Fig. 2(a).

The relative phase $\varphi$ can be imprinted, e.g., optically, via tilting of the apparatus, or via induced interatomic interactions. For example, if the trap is briefly tilted at $T_{\text {rev }} / 2$ the gravitational potential yields the phase $\varphi_{g} \approx 2 m g R t_{\mathrm{d}} \sin \theta / \hbar$, where $\theta$ is the tilt angle and $t_{\mathrm{d}}$ is the revival lifetime. The latter is the dispersion timescale $t_{\mathrm{d}} \approx 1 / \omega_{\perp}$ of the initial wave packet of width $\sqrt{\hbar / \omega_{\perp} m}$. For the above example, this requires tilting with a precision of hundreds of microradians.

Likewise, if the magnetic field on one side of the ring is detuned from the zero crossing of the Feshbach resonance, the matter wave acquires a relative phase $\varphi_{a} \approx 4 \pi \hbar a n_{\mathrm{BEC}} t_{\mathrm{d}} / m$, where $a$ is the induced scattering length and $n_{\mathrm{BEC}}$ is the particle density in the initial state. With this one can measure the scattering length with precision $\Delta a \approx 0.2 a_{0}$ (with $a_{0}$ the Bohr radius), on par with state-of-the-art time-of-flight [42] and spectroscopic [43] measurements for ${ }^{39} \mathrm{~K}$.

Conclusions. We introduced orbital angular momentum interference as an attractive platform for trapped matter-wave interferometry in toroidal geometries. Since the proposed scheme relies on the universal property of orbital momentum quantization, realizations with many different systems can be readily envisioned, e.g., single atoms or BECs in optical traps, ions in electric traps, electrons in solid state quantum rings, as well as molecules and nanoparticles in optical or electrical traps. For the case of a BEC in an optical trap, we have shown that the protocol is feasible with present-day technology.

The interference effect is sensitive to the presence of gauge fields. In the presence of a magnetic field flux $\Phi$, for instance, the revival of particles with charge $q$ will be displaced by the angle $q \Phi / \hbar$. Assuming that displacements on the size of the initial wave packet can be angularly resolved, fields below $10^{-7} \mathrm{~T}$ level can be detected with the setup described above.

Acknowledgments. We thank Markus Arndt, Thorsten Schumm, and Philipp Haslinger for helpful discussions. F.K. acknowledges support by the Austrian Science Fund (FWF) Project No. W1210-N25, B.A.S. acknowledges funding from the European Union's Horizon 2020 research and innovation programme under the Marie Skłodowska-Curie grant agreement No. 841040.
[1] Y.-J. Wang, D. Z. Anderson, V. M. Bright, E. A. Cornell, Q. Diot, T. Kishimoto, M. Prentiss, R. A. Saravanan, S. R. Segal, and $\mathrm{S}$. Wu, Atom Michelson Interferometer on a Chip Using a Bose-Einstein Condensate, Phys. Rev. Lett. 94, 090405 (2005).

[2] O. Garcia, B. Deissler, K. J. Hughes, J. M. Reeves, and C. A. Sackett, Bose-Einstein-condensate interferometer with macroscopic arm separation, Phys. Rev. A 74, 031601(R) (2006).

[3] M. Horikoshi and K. Nakagawa, Suppression of Dephasing Due to a Trapping Potential and Atom-Atom Interactions in a Trapped-Condensate Interferometer, Phys. Rev. Lett. 99, 180401 (2007).

[4] M. Karski, L. Förster, J.-M. Choi, A. Steffen, W. Alt, D. Meschede, and A. Widera, Quantum walk in position space with single optically trapped atoms, Science 325, 174 (2009).

[5] T. Berrada, S. van Frank, R. Bücker, T. Schumm, J.-F. Schaff, and J. Schmiedmayer, Integrated Mach-Zehnder interferom- eter for Bose-Einstein condensates, Nat. Commun. 4, 2077 (2013).

[6] B. Rauer, S. Erne, T. Schweigler, F. Cataldini, M. Tajik, and J. Schmiedmayer, Recurrences in an isolated quantum many-body system, Science 360, 307 (2018).

[7] E. Urban, N. Glikin, S. Mouradian, K. Krimmel, B. Hemmerling, and H. Haeffner, Coherent Control of the Rotational Degree of Freedom of a Two-Ion Coulomb Crystal, Phys. Rev. Lett. 123, 133202 (2019).

[8] V. Xu, M. Jaffe, C. D. Panda, S. L. Kristensen, L. W. Clark, and H. Müller, Probing gravity by holding atoms for 20 seconds, Science 366, 745 (2019).

[9] O. Zobay and B. M. Garraway, Two-Dimensional Atom Trapping in Field-Induced Adiabatic Potentials, Phys. Rev. Lett. 86, 1195 (2001). 
[10] T. A. Bell, J. A. P. Glidden, L. Humbert, M. W. J. Bromley, S. A. Haine, M. J. Davis, T. W. Neely, M. A. Baker, and H. Rubinsztein-Dunlop, Bose-Einstein condensation in large time-averaged optical ring potentials, New J. Phys. 18, 035003 (2016).

[11] J. L. Ville, T. Bienaimé, R. Saint-Jalm, L. Corman, M. Aidelsburger, L. Chomaz, K. Kleinlein, D. Perconte, S. Nascimbène, J. Dalibard, and J. Beugnon, Loading and compression of a single two-dimensional Bose gas in an optical accordion, Phys. Rev. A 95, 013632 (2017).

[12] S. Pandey, H. Mas, G. Drougakis, P. Thekkeppatt, V. Bolpasi, G. Vasilakis, K. Poulios, and W. von Klitzing, Hypersonic BoseEinstein condensates in accelerator rings, Nature (London) 570, 205 (2019).

[13] S. Gupta, K. W. Murch, K. L. Moore, T. P. Purdy, and D. M. Stamper-Kurn, Bose-Einstein Condensation in a Circular Waveguide, Phys. Rev. Lett. 95, 143201 (2005).

[14] C. Ryu, M. F. Andersen, P. Cladé, V. Natarajan, K. Helmerson, and W. D. Phillips, Observation of Persistent Flow of a BoseEinstein Condensate in a Toroidal Trap, Phys. Rev. Lett. 99, 260401 (2007).

[15] A. Ramanathan, K. C. Wright, S. R. Muniz, M. Zelan, W. T. Hill, C. J. Lobb, K. Helmerson, W. D. Phillips, and G. K. Campbell, Superflow in a Toroidal Bose-Einstein Condensate: An Atom Circuit With a Tunable Weak Link, Phys. Rev. Lett. 106, 130401 (2011).

[16] A. Das, J. Sabbatini, and W. H. Zurek, Winding up superfluid in a torus via Bose-Einstein condensation, Sci. Rep. 2, 352 (2012).

[17] S. Eckel, J. G. Lee, F. Jendrzejewski, N. Murray, C. W. Clark, C. J. Lobb, W. D. Phillips, M. Edwards, and G. K. Campbell, Hysteresis in a quantized superfluid 'atomtronic' circuit, Nature (London) 506, 200 (2014)

[18] S. Eckel, A. Kumar, T. Jacobson, I. B. Spielman, and G. K. Campbell, A Rapidly Expanding Bose-Einstein Condensate: An Expanding Universe in the Lab, Phys. Rev. X 8, 021021 (2018).

[19] G. E. Marti, R. Olf, and D. M. Stamper-Kurn, Collective excitation interferometry with a toroidal Bose-Einstein condensate, Phys. Rev. A 91, 013602 (2015).

[20] S. Ragole and J. M. Taylor, Interacting Atomic Interferometry for Rotation Sensing Approaching the Heisenberg Limit, Phys. Rev. Lett. 117, 203002 (2016).

[21] J. L. Helm, T. P. Billam, A. Rakonjac, S. L. Cornish, and S. A. Gardiner, Spin-Orbit-Coupled Interferometry with RingTrapped Bose-Einstein Condensates, Phys. Rev. Lett. 120, 063201 (2018).

[22] R. W. Robinett, Quantum wave packet revivals, Phys. Rep. 392, 1 (2004).

[23] T. Seideman, Revival Structure of Aligned Rotational Wave Packets, Phys. Rev. Lett. 83, 4971 (1999).

[24] M. Spanner, E. A. Shapiro, and M. Ivanov, Coherent Control of Rotational Wave-Packet Dynamics Via Fractional Revivals, Phys. Rev. Lett. 92, 093001 (2004).

[25] M. D. Poulsen, E. Peronne, H. Stapelfeldt, C. Z. Bisgaard, S. S. Viftrup, E. Hamilton, and T. Seideman, Nonadiabatic alignment of asymmetric top molecules: Rotational revivals, J. Chem. Phys. 121, 783 (2004).

[26] A. S. Moskalenko, A. Matos-Abiague, and J. Berakdar, Revivals, collapses, and magnetic-pulse generation in quantum rings, Phys. Rev. B 74, 161303(R) (2006).
[27] B. A. Stickler, B. Papendell, S. Kuhn, B. Schrinski, J. Millen, M. Arndt, and K. Hornberger, Probing macroscopic quantum superpositions with nanorotors, New J. Phys. 20, 122001 (2018).

[28] P. Navez, S. Pandey, H. Mas, K. Poulios, T. Fernholz, and W. von Klitzing, Matter-wave interferometers using TAAP rings, New J. Phys. 18, 075014 (2016).

[29] G. Pelegrí, J. Mompart, and V. Ahufinger, Quantum sensing using imbalanced counter-rotating Bose-Einstein condensate modes, New J. Phys. 20, 103001 (2018).

[30] V. M. Fomin, Physics of Quantum Rings (Springer, Berlin, 2013).

[31] J. Millen, T. S. Monteiro, R. Pettit, and A. N. Vamivakas, Optomechanics with levitated particles, Rep. Prog. Phys. 83, 026401 (2020).

[32] G. A. Vugalter, A. K. Das, and V. A. Sorokin, A charged particle on a ring in a magnetic field: Quantum revivals, Eur. J. Phys. 25, 157 (2003).

[33] A. Lorke, R. J. Johannes Luyken, A. O. Govorov, J. P. Kotthaus, J. M. Garcia, and P. M. Petroff, Spectroscopy of Nanoscopic Semiconductor Rings, Phys. Rev. Lett. 84, 2223 (2000).

[34] Y. Aharonov and A. Casher, Topological Quantum Effects for Neutral Particles, Phys. Rev. Lett. 53, 319 (1984).

[35] H. Wei, R. Han, and X. Wei, Quantum Phase of Induced Dipoles Moving in a Magnetic Field, Phys. Rev. Lett. 75, 2071 (1995).

[36] P. Leboeuf and N. Pavloff, Bose-Einstein beams: Coherent propagation through a guide, Phys. Rev. A 64, 033602 (2001).

[37] S. Schwartz, M. Cozzini, C. Menotti, I. Carusotto, P. Bouyer, and S. Stringari, One-dimensional description of a BoseEinstein condensate in a rotating closed-loop waveguide, New J. Phys. 8, 162 (2006).

[38] C. Chin, R. Grimm, P. Julienne, and E. Tiesinga, Feshbach resonances in ultracold gases, Rev. Mod. Phys. 82, 1225 (2010).

[39] C. S. Bederián and A. D. Dente, Boosting quantum evolutions using Trotter-Suzuki algorithms on GPUs, in Proceedings of HPCLatAm-11, 4th High-Performance Computing Symposium, edited by N. Wolovick and G. Hernández (Sociedad Argentina de Informática (SADIO), Buenos Aires, 2011), pp. 63-75.

[40] P. Wittek and F. M. Cucchietti, A second-order distributed Trotter-Suzuki solver with a hybrid CPU-GPU kernel, Comput. Phys. Commun. 184, 1165 (2013).

[41] P. Wittek and L. Calderaro, Extended computational kernels in a massively parallel implementation of the Trotter-Suzuki approximation, Comput. Phys. Commun. 197, 339 (2015).

[42] C. D’Errico, M. Zaccanti, M. Fattori, G. Roati, M. Inguscio, G. Modugno, and A. Simoni, Feshbach resonances in ultracold ${ }^{39}$ K, New J. Phys. 9, 223 (2007).

[43] S. Falke, H. Knöckel, J. Friebe, M. Riedmann, E. Tiemann, and C. Lisdat, Potassium ground-state scattering parameters and Born-Oppenheimer potentials from molecular spectroscopy, Phys. Rev. A 78, 012503 (2008).

Correction: $2 \gamma$ and $2 q$ have been replaced by $\gamma$ and $q$ in Eq. (3) and in the paragraph below it, as well as in the caption to Figure 1 and in the paragraph above the Acknowledgment section. The previously published Figure 1 also has been replaced. Minor errors have also been fixed in Eqs. (3), (10), and (11) and in text that appears two paragraphs above Eq. (3). 